GI-MS47-05

\section{Crystallographic software for the next generation}

Richard Cooper

1. Chemical Crystallography Lab, Department of Chemistry, University of Oxford, Oxford, United Kingdom

email: richard.cooper@chem.ox.ac.uk

Where can crystallographic computing take you in your career and what can you do about it? I will reflect on some advances and side-steps taken through a career where crystallographic software has been the common feature.

In your early career you will be learning useful skills, staying at the forefront of the field and keeping your options open for your next move. Developing crystallographic software can give you transferable skills and an in-depth knowledge of the fundamentals of the subject. With these skills, many crystallographic software developers move between quite different scientific problems during their careers.

I had no experience of crystallography or software development when I started an undergraduate research project developing methods for visualising and interacting with crystallographic data. I will present some lessons learned along the way and how I might plan and do things differently with the benefit of hindsight.

Keywords: software, careers, crystallography
GI-MS48 Education in crystallography

Chairs: Prof. Mike Glazer, Dr. Aleksandar Visnjevac

GI-MS48-01

\section{Ask not what crystallography can do for you - ask what you can do for crystallography}

Chiara Massera $^{1}$, Marijana Đaković ${ }^{2}$, Annalisa Guerri $^{3}$, Catharine Esterhuysen $^{4}$, Serena Chiara Tarantino ${ }^{5}$

1. University of Parma, Parma, Italy

2. University of Zagreb, Zagreb, Croatia

3. University of Florence, Florence, Italy

4. Stellenbosch University, Stellenbosch, South Africa

5. University of Pavia, Pavia, Italy

email: chiara.massera@unipr.it

Promoting and spreading crystallography at all levels of education: that is the aim of GIG3 - The General Interest Group on Education in Crystallography - launched at the ECM in Basel in September 2016. The main objectives of GIG3 are the creation of a network for the efficient coordination of common activities and of new projects, and ultimately increasing the awareness of the general public about the existence, utility and versatility of crystallography as a field of study and application.

In this session some of the past and ongoing activities of the Group will be outlined and, more importantly, ideas and suggestions will be discussed on how to transform the Group's tasks into concrete initiatives.

Keywords: teaching, promotion, dissemination 